\title{
Systematic Comparison of Ultraviolet Photodissociation and Electron Transfer Dissociation for Peptide Anion Characterization
}

\author{
Jared B. Shaw, ${ }^{1}$ James A. Madsen, ${ }^{1}$ Hua Xu, ${ }^{2}$ Jennifer S. Brodbelt ${ }^{1}$ \\ ${ }^{1}$ Department of Chemistry and Biochemistry, The University of Texas at Austin, Austin, TX 78712, USA \\ ${ }^{2}$ Center for Proteomics and Bioinformatics, Case Western Reserve University, Cleveland, OH 44106, USA
}

\begin{abstract}
Ultraviolet photodissociation at $193 \mathrm{~nm}$ (UVPD) and negative electron transfer dissociation (NETD) were compared to establish their utility for characterizing acidic proteomes with respect to sequence coverage distributions (a measure of product ion signals across the peptide backbone), sequence coverage percentages, backbone cleavage preferences, and fragmentation differences relative to precursor charge state. UVPD yielded significantly more diagnostic information compared with NETD for lower charge states $(n \leq 2)$, but both methods were comparable for higher charged species. While UVPD often generated a more heterogeneous array of sequence-specific products $\left(b-, y-, c_{-}, z-, Y\right.$ -,$d$-, and $w$-type ions in addition to $a$ - and $x$ type ions), NETD usually created simpler sets of $a / x$-type ions. LC-MS/UVPD and LC-MS/NETD analysis of protein digests utilizing high $\mathrm{pH}$ mobile phases coupled with automated database searching via modified versions of the MassMatrix algorithm was undertaken. UVPD generally outperformed NETD in stand-alone searches due to its ability to efficiently sequence both lower and higher charge states with rapid activation times. However, when combined with traditional positive mode CID, both methods yielded complementary information with significantly increased sequence coverage percentages and unique peptide identifications over that of just CID alone.
\end{abstract}

Key words: Tandem mass spectrometry, Protein identification, Negative electron transfer dissociation, Ultraviolet photodissociation

\section{Introduction}

Standard proteomics experiments typically involve a $\mathcal{N}$ "bottom-up" strategy consisting of protein digestion, liquid chromatographic separation using acidic eluents, ionization in the positive mode, tandem mass spectrometry (MS) via collision induced dissociation (CID) [1, 2], and

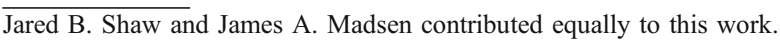

Electronic supplementary material The online version of this article (doi:10.1007/s13361-012-0424-9) contains supplementary material, which is available to authorized users.

Correspondence to: Jennifer S. Brodbelt; e-mail: jbrodbelt@cm.utexas.edu automated database searching [3, 4]. This general protocol consistently works well for the analysis of peptides cations [5]. In addition, alternative dissociation methods, such as electron capture/transfer dissociation [6-11] as well as infrared photodissociation (IRMPD) [12-15] and ultraviolet photodissociation (UVPD [16-27] have been developed to supplement CID. While CID remains the most popular MS/ MS method for peptide cations, it often falls short for the characterization of more acidic species, such as peptides containing several acidic amino acids and/or acidic post translational modifications (PTM), due to low ion signals and poor MS/MS patterns. Acidic peptides ionize readily in the negative mode via deprotonation [28, 29]; however, CID of peptide anions generally yields low sequence coverage, 
high abundances of uninformative neutral losses, and overall unpredictable fragmentation patterns [30-32]. It is now well recognized that many important modifications such as phosphorylation, sulfonation, acetylation, and glycosylation render proteins more acidic after translation [33]. Moreover, all proteomes exhibit a bimodal distributions of protein isoelectric points (pI) [34-37]. In other words, every proteome contains acidic and basic subsets of proteins with very few proteins possessing a $\mathrm{pI}$ close to cellular $\mathrm{pH}[34$ 37]. As a result of these two factors, there is a need for improved methods for the characterization of the acidic regions of proteins as well as the acidic subset of proteins, including those approaches specifically adapted for analysis of peptide anions.

Several MS/MS and $\mathrm{MS}^{\mathrm{n}}$ methods have recently been developed for analysis of peptide anions to combat the downfalls of conventional CID [38-44]. The majority of these techniques have utilized electrons or photons to promote primarily $\mathrm{C}_{\alpha}-\mathrm{C}(\mathrm{O})$ bond cleavages, resulting in $a$ and $x$-type sequence ions. For example, the interaction of peptides with high-energy electrons, termed electron detachment dissociation (EDD), has been shown to be useful for sequencing peptide anions [38, 39]. Activated-electron photodetachment dissociation (a-EPD), an MS anion activation method, uses UV irradiation at $262 \mathrm{~nm}$ in the first step to produce charge-reduced peptide radicals, which are subsequently dissociated by collisional activation in the second step [40, 45]. EDD and a-EPD were compared recently for characterization of peptide anions, and while both techniques yielded mostly the same types of ions $(a / x$ type), the abundances were significantly different [40]. Cleavages next to aromatic and histidine residues were favored for a-EPD, whereas cleavages next to negative charge solvation sites on the backbone were preferential for EDD [40]. Recently, a method involving high kinetic energy metastable ions (MAD-MS) was used to dissociate phosphorylated and sulfonated peptide anions; $\mathrm{C}_{\alpha}-\mathrm{C}(\mathrm{O})$ bond cleavages were again the main dissociation channels [41]. The development of EDD, a-EPD, and MAD-MS all represent promising advances for peptide anion characterization, but these methods generally afford low dissociation efficiencies and thus require long averaging times to generate interpretable spectra $[40,41]$. Also, implementing these methods on liquid chromatographic timescales is problematic because the required activation times often range from half a second up to multiple seconds for peptide dissociation [39, 45]. Two alternative anion dissociation techniques that require less averaging and lower activation times to produce reasonable spectra, therefore allowing more facile integration with LC-MS/MS workflows, are negative electron transfer dissociation (NETD) [42, 43] and $193 \mathrm{~nm}$ ultraviolet photodissociation (UVPD) [44]. The former involves the reaction of peptide anions with electrondeficient radical cations, causing electron transfer from the peptide to the reagent that results in backbone dissociation to create mainly $a$ and $x$ ions [42,43]. This method has shown great potential in sequencing peptides in high-throughput experiments [46]; $193 \mathrm{~nm}$ UVPD delivers high-energy $(6.4 \mathrm{eV})$ photons to peptides and has been shown to yield diagnostic spectra dominated by $a$ and $x$ ions and utilizing activation times as little as $5 \mathrm{~ns}$ (in combination with LC-MS/ MS) [44]. UVPD has more recently been used in conjunction with automated database searching and sequencing of peptide anions for the analysis of complex protein samples [47]. Since both NETD and UVPD have been promising for the sensitive and high-throughput analysis of acidic proteomes, we have undertaken a comparison of NETD and UVPD for the characterization of a series of modified (e.g., phosphorylated) and unmodified peptide anions. The sequence coverage distribution (a measure of product ion signals across the peptide backbone), sequence coverage percentages, backbone cleavage preferences, and fragmentation differences with respect to precursor charge state were assessed between the methods. Results from the analysis of a seven protein mixture via automated MassMatrix [48-50] interpretation and LCMS/MS are also presented.

\section{Experimental}

\section{Materials and Sample Preparation}

All peptides were purchased from AnaSpec (Fremont, CA, USA), and all proteins were purchased from Sigma Aldrich (St. Louis, MO, USA) except for chicken lysozyme C, which was purchased from MP Biomedicals, LLC (Solon, OH, USA). All peptides and proteins were used without further purification. HPLC grade methanol and water (Fisher Scientific, Pittsburg, PA, USA) were used for preparing peptide working solutions. HPLC grade ammonium hydroxide and argon of $\geq 99.998 \%$ purity were purchased from Sigma Aldrich (St. Louis, MO, USA). All optical components were purchased from Edmund Optics Inc. (Barrington, NJ, USA).

An equimolar mixture of bovine $\alpha$-casein, $\beta$-casein, $\beta$ lactoglobulin A, BSA, ribonuclease A, and chicken lysozyme $\mathrm{C}$ was made in $100 \mathrm{mM}$ sodium bicarbonate. Two hundred $\mu \mathrm{g}$ of total protein was reduced with dithiothreitol (DTT) for $30 \mathrm{~min}$, and then incubated with iodoacetamide at $40{ }^{\circ} \mathrm{C}$ for $1 \mathrm{~h}$ in the dark. Excess iodoacetamide was quenched with additional DTT. Four $\mu \mathrm{L}$ of $1 \mu \mathrm{g} / \mu \mathrm{L}$ trypsin in $1 \mathrm{mM} \mathrm{HCl}$ was added, giving a protein to enzyme ratio of 50:1. The digestion solution was incubated overnight at $37^{\circ} \mathrm{C}$. The digest was desalted using $\mathrm{C} 18$ cartridges then dried and reconstituted in water to a concentration of $1 \mu \mathrm{g} / \mu \mathrm{L}$.

\section{Instrumentation}

A Bruker Daltonics (Billerica, MA, USA) HCT Ultra ETD II ion trap mass spectrometer was modified to allow the introduction of $193 \mathrm{~nm}$ photons into the trapping volume. Briefly, two $25 \mathrm{~mm} \mathrm{CaF}_{2}$ windows were mounted in the vacuum manifold lid above the ion trap. The laser beam was 
passed through $2 \mathrm{~mm}$ holes in the ring electrode (oriented vertically) of the quadrupole ion trap. Broadband deep UV enhanced $12.5 \mathrm{~mm}$ mirrors were placed in $45^{\circ}$ mounts under and next to the ion trap to reflect the laser beam back out of the mass spectrometer. This configuration allowed facile alignment of the laser through the system by monitoring the intensity and profile of the beam exiting the system. Ultraviolet photons (193 nm, $5 \mathrm{~ns}$ pulse, $500 \mathrm{~Hz}, 8 \mathrm{~mJ} /$ pulse max) were generated by an ExciStar XS 500 laser (Coherent Inc., Santa Clara, CA, USA) and used without focusing or collimating the beam. An external trigger supplied through the auxiliary interface port gated a pulse/delay generator (model 505; Berkely Nucleonics Corp., San Rafael, CA, USA), which triggered the laser during the fragmentation period of the ion trap scan function (two pulses total). An override in the instrument user interface and replacement of the methane carrier gas with argon allowed efficient production of fluoranthene radical cations by argon chemical ionization and transmission of the radical cations to the ion trap. A reagent crucible temperature of $60{ }^{\circ} \mathrm{C}$, a filament current of $1 \mu \mathrm{A}$, and electron energy of $70 \mathrm{eV}$ were used.

\section{Mass Spectrometry and Liquid Chromatography}

Ten $\mu \mathrm{M}$ peptide working solutions composed of 70/30 (vol/ vol) methanol/water with $0.1 \%$ ammonium hydroxide were infused at $1.5 \mu \mathrm{L} / \mathrm{min}$ for electrospray ionization. NETD experiments were performed with a low mass cutoff of $\mathrm{m} / \mathrm{z}$ 160,10 to $15 \mathrm{~ms}$ of reagent accumulation, and 150 to $250 \mathrm{~ms}$ reaction time. UPVD experiments were performed with $4 \mathrm{~ms}$ activation periods allowing two $4 \mathrm{~mJ}$ laser pulses and a $\mathrm{q}_{\mathrm{z}}$-value of 0.110 , which allowed detection of low $\mathrm{m} / \mathrm{z}$ product ions.

LC-MS/MS experiments were performed with a DionexUltiMate 3000 system (Sunnyvale, CA) using a capillary flowsplitter. An Agilent ZORBAX 300Extend-C18 column (Santa Clara, CA, USA) $(150 \times 0.3 \mathrm{~mm}, 3.5 \mu \mathrm{m}$ particle size) was used for all separations. The column temperature was held at $25{ }^{\circ} \mathrm{C}$. For negative ion mode experiments (NETD and UVPD), eluents $\mathrm{A}$ and $\mathrm{B}$ consisted of $0.05 \%$ ammonium hydroxide in water and acetonitrile, respectively. A 120-min gradient consisting of the first $5 \mathrm{~min}$ at $3 \%$ eluent B and a linear increase to $35 \%$ eluent B over $120 \mathrm{~min}$ at a flow rate of $4 \mathrm{uL} / \mathrm{min}$ was used to separate the protein mixture digest. CID experiments were performed in positive mode and utilized $0.1 \%$ formic acid in water and acetonitrile for eluents $\mathrm{A}$ and $\mathrm{B}$, respectively. The same gradients were used for positive mode except the gradient ended at $40 \%$ eluent B. $5 \mu \mathrm{L}$ of $1 \mu \mathrm{g} / \mu \mathrm{L}$ protein mixture digest was injected on column. Data-dependent acquisition consisted of a survey scan of $m / z 400-2000$ followed by five MS/MS scan events for all activation methods. For LC-MS/ NETD, the precursor charge state preference was set to exclude singly charged ions. No precursor charge state preference was specified for LC-MS/UVPD. A preference for doubly charged ions was utilized for LC-MS/CID experiment. For all LC-MS/MS experiments, eight averages were used for all MS/MS events and dynamic exclusion was set to one repeat and exclude for $60 \mathrm{~s}$.

MassMatrix was used for all database searching [48-50]. This algorithm, which was originally developed for positive mode CID, was modified to allow searching of negative mode MS/MS data by both UVPD and NETD. The methods were optimized individually based on the specific fragmentation behavior associated with each method. For example, NETD searches were performed with several forms of $a$ - and $x$-type ions (e.g., $a / x \pm 1$ due to hydrogen migration), and therefore searching scores are elevated compared with UVPD, which uses only one form of $a$ - and $x$ - ions. Preferential cleavages at proline, glutamic acid, and aspartic acid that yield specific Y- and y-type ions, and $c / z$ ions are also included in searches for UVPD [44].

Using these modified MassMatrix algorithms, LC-MS/ MS data sets were searched against the cow International Protein Index (IPI v3.73, Sept 27, 2011) modified to include the chicken lysozyme $\mathrm{C}$ sequence using the MassMatrix database search engine v2.4.0. LC-MS/MS were performed in triplicate, and the data from the replicate experiments were merged prior to database searching. Search parameters utilized were trypsin, two missed cleavages, phosphorylation of $\mathrm{S}$ and $\mathrm{T}$ as variable modifications, iodoacetamide/ carbamidomethylation of $\mathrm{C}$ as a fix modification, precursor ion tolerance of $2 \mathrm{Da}$, and a product ion tolerance of $1 \mathrm{Da}$. Peptide hits were filtered by $\mathrm{pp}$ and $\mathrm{pp}_{2}$ scores less than 5 and $\mathrm{pp}_{\text {tag }}$ scores less than 0.5 [48] for which the $\mathrm{pp}$ and $\mathrm{pp} 2$ scores are a statistical measure of the number of matched product ions and the total abundance of matched product ions, respectively. All suspect peptide spectral matches (PSMs) were manually verified.

\section{Results and Discussion}

Figure 1 shows NETD ( $a$ and $c$ ), and UVPD (b and d) spectra of doubly and triply deprotonated KPLLIIAEDVEGEY. NETD of the doubly charged peptide results in production of eight $x$-ions and two $a$-ions, corresponding to ten out of 13 possible backbone cleavage sites. The dominant dissociation pathways include the neutral loss of $\mathrm{CO}_{2}$ and non-dissociative electron transfer (NETnoD), the latter yielding intact charge-reduced precursor ions (labeled as $[\mathrm{M}-2 \mathrm{H}]^{-\bullet}$ ). The most abundant sequence-informative products are the $x_{10}, x_{11}, x_{12}$, and $x_{13}$ ions. A fluoranthene adduct of the intact precursor is also observed in all NETD spectra and is denoted by the label $\Delta$ in Figure 1a and c. The $\mathrm{m} / \mathrm{z}$ value of the fluoranthene adduct species can be accurately predicted based on the consistent mass shift of $202 \mathrm{Da}$ and thus can be subtracted from the spectra prior to the database searches. UVPD of the doubly charged species yields an extensive array of sequence-informative product ions. Whereas NETD exclusively produced $a$ - and $x$-type product ions, UVPD yields $b-, y-, c-, z-, Y-, d$-, and $w$-type product ions in addition to the dominant $a$ - and $x$-ions. The large array of product ions upon UVPD yielded $100 \%$ 


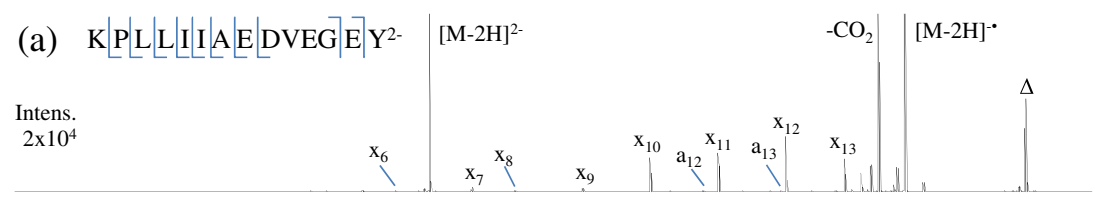

(b) KLPLLLIIAEDVEGGE $\mathrm{Y}^{2-}$

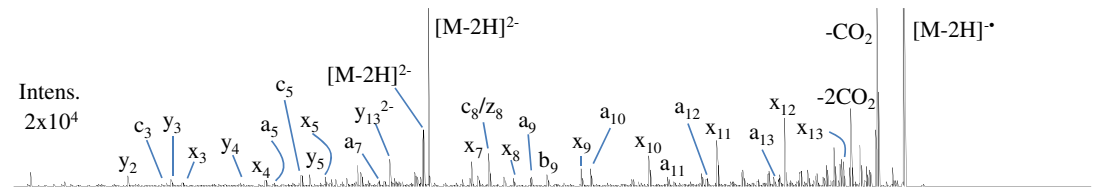

(c) KLPLLLILAE D V E E E E Y3-

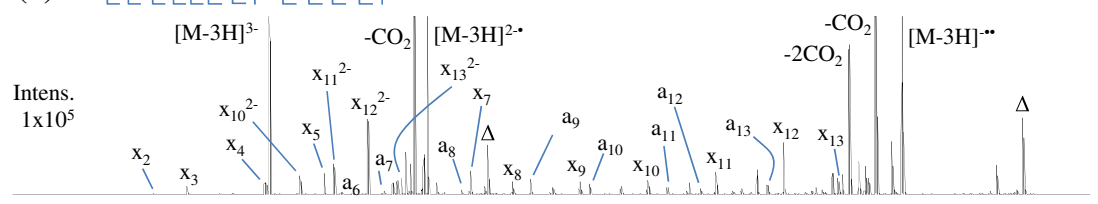

(d) K|PLLLIIIAE D V E G E $\mathrm{Y}^{3-}$

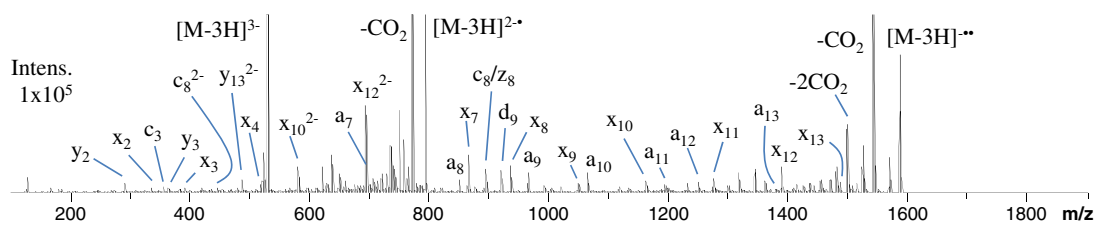

Figure 1. NETD (a) and (c) and UVPD (b) and (d) spectra of doubly and triply charged KPLLIIAEDVEGEY. $\Delta$ indicates fluoranthene adduction to the precursor

sequence coverage as well as complementary $\mathrm{N}$ - and $\mathrm{C}$ terminal product ions (i.e., $a / x$ product ion pairs) for nine of the 13 backbone cleavage sites. UVPD also promotes the loss of $\mathrm{CO}_{2}$ from the precursor as well as charge-reduction by photo-induced electron detachment, a pathway noted in our previous UVPD study of peptide anions [44] NETD of the triply charged peptide in Figure 1c provides complete sequence coverage and complementary $a$ - and $x$-ions for seven of the 13 peptide backbone cleavage sites. The triply charged peptide yielded five additional $a$-ions and four $x$ ions relative to the doubly charged peptide. Although the $a / x$ ion series has higher abundance and affords greater coverage than observed for the corresponding doubly charged peptide, the loss of $\mathrm{CO}_{2}$ and NETnoD remain the most dominant pathways. The fragmentation pattern produced by UVPD of the triply charged species is similar to that of the doubly charged species. Complete sequence coverage is achieved, and complementary $a / x$ ion pairs are observed for nine of the 13 peptide backbone cleavage sites.

For the peptide KPLLIIAEDVEGEY, charge reduction accounts for $59 \%$ and $42 \%$ of product ions (based on peak area) produced by NETD of the 2- and 3- species, respectively. Similarly, UVPD resulted in $31 \%$ and $30 \%$ charge reduction for the 2- and 3- species, respectively. Neutral loss of $\mathrm{CO}_{2}$ from the precursor accounts for $24 \%$ and $34 \%$ of the product ions upon NETD and $15 \%$ and $25 \%$ upon UVPD for the 2- and 3- species, respectively. UVPD produced less extensive charge reduction and $\mathrm{CO}_{2}$ loss from the precursor than NETD; however small molecule neutral losses $\left(\mathrm{CO}_{2}, \mathrm{NH}_{3}\right.$ and $\left.\mathrm{H}_{2} \mathrm{O}\right)$ from sequence ions were more notable upon UPVD. NETD produced only one detectable neutral loss sequence ion $\left(\mathrm{x}_{7}-\mathrm{CO}_{2}\right)$ for the doubly charged peptide. The loss of $\mathrm{CO}_{2}$ loss was observed from the $\mathrm{x}_{7}, \mathrm{x}_{9}, \mathrm{x}_{10}, \mathrm{x}_{11}, \mathrm{x}_{12}$, and $\mathrm{x}_{13}$ ions upon NETD of the triply charged peptide, as well as ammonia loss from $\mathrm{a}_{13}$. $\mathrm{CO}_{2}, \mathrm{NH}_{3}$ and $\mathrm{H}_{2} \mathrm{O}$ losses occurred for a majority of the sequence ions upon UVPD, with even multiple neutral losses in some cases. This outcome is attributed to the energetics of NETD versus UVPD. Polfer and coworkers estimated the recombination energy of electron transfer with fluoranthene radical cations to be $2.4-4.5 \mathrm{eV}$ depending on the site of electron abstraction [42]. In the same study, they also reported that the more energetic electron abstraction by Xe radical cations (estimated recombination energy of 6.7$8.7 \mathrm{eV}$ ) vastly increased the number of fragment ions observed [42]. However, the additional fragment ions mostly corresponded to sequential neutral losses from $a^{\bullet}$ and $x$-ions instead of new diagnostic products arising from different fragmentation channels (i.e., b, c, y, z-ions) [42]. The greater energy deposition upon absorption of a single $193 \mathrm{~nm}$ photon $(6.4 \mathrm{eV})$ and wider array of fragmentation channels accessible compared with electron abstraction by fluoranthene radical cations accounts for the larger array of product ion types as well as the greater extent of neutral losses. UVPD product ions may also possess greater internal energy upon formation, thus contributing to more extensive secondary dissociation and thus elevated levels of neutral losses compared to NETD. 
The NETD (a and c) and UVPD (b and d) spectra of the doubly and triply deprotonated phosphopeptide TSTEPQyQPGNEL are illustrated in Figure 2. The phosphorylation site can be pinpointed to the tyrosine residue based on the NETD and UVPD spectra for both the doubly and triply charged species. However, for the doubly charged species, UVPD affords greater confidence in the identification of the sequence and the localization of the phosphorylation site as a result of the production of peptide backbone cleavages both $\mathrm{N}$ terminal and $\mathrm{C}$-terminal to the phosphorylated residue and complete sequence coverage of the rest of the peptide. NETD did not result in the loss of the phosphate group from the precursor or from any of the sequence ions. Importantly, UVPD produced $\mathrm{H}_{2} \mathrm{PO}_{4}^{-}$, and $\mathrm{PO}_{3}^{-}$reporter ions that can be used to confirm peptide phosphorylation in bottom-up proteomic experiments. The UPVD spectrum of singly charged TSTEPQyQPGNEL is shown in Supplemental Figure 1. UVPD of the singly charged species resulted in nearly complete series of $a$ and $x$ ions as well as confident assignment of the site of phosphorylation. In this case, the phosphate reporter ions fall below the low mass cutoff and are not detected, but there is an abundant phosphate neutral loss from the precursor. In general, the UVPD spectrum for the singly deprotonated phosphopeptide is more congested with internal ions and neutral losses compared to the UVPD mass spectra obtained for the corresponding doubly and triply charged species.

Since activation/dissociation by NETD is more highly dependent on the peptide charge state than UVPD, a comparison of NETD and UVPD was undertaken for six doubly and triply deprotonated peptides. Sequence coverage distributions were calculated for each peptide and charge state based on the summed peak areas of complementary $a / x$ ion pairs normalized to the most abundant $a / x$ ion pair. Only product ions with $\mathrm{S} / \mathrm{N} \geq 3$ were included in the sequence coverage distributions. The results are shown in Figure 3. In general, NETD performs poorly for the doubly deprotonated peptides as witnessed by the rather sparse bar graphs in Figure 3. For these cases, the most dominant pathways produced $x$-ions corresponding to cleavage near the $\mathrm{N}$-terminus with very few low abundance $a$-ions. In fact, there are zero complementary $a$ - and $x$-ions pairs detected for the doubly charged precursors, signifying notable localization of the charge sites upon NETD. NETD of the triply deprotonated species yields greater abundances and broader distributions of backbone cleavages compared to the doubly charged species, although still occurring with notable gaps in coverage. UVPD produced substantially broader distributions of peptide backbone cleavages for both the doubly and triply charged species of all the peptides analyzed. For the doubly charged species, UVPD produced greater product ion abundances for nearly every possible peptide backbone cleavage site compared with NETD. This is partially attributed to the production of complementary $a$ - and $x$-ion pairs for most backbone cleavage sites upon UVPD, whereas NETD predominantly produced $x$ ions. Table 1 summarizes the sequence coverages obtained by NETD and UVPD for the same six peptides in the 2- and 3charge states. UVPD produced $100 \%$ sequence coverage for all the peptides (both doubly and triply charged species). NETD produced average sequence coverage of $37 \%$ for the doubly charged species and $83 \%$ for the triply charged species.

To enable a more extensive comparison of the performance of NETD and UVPD for protemoics-type experiments, as well as benchmarking these MS/MS methods relative to conventional positive mode CID, LC-MS/NETD

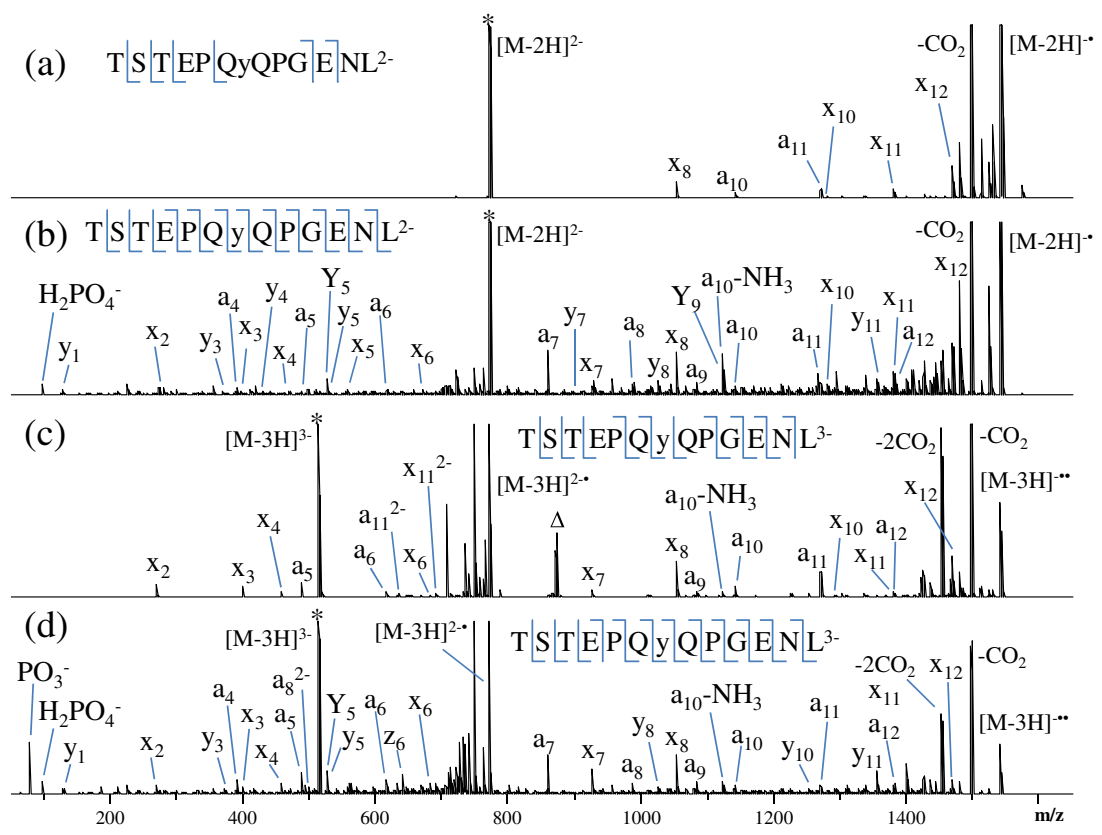

Figure 2. NETD (a) and (c) and UVPD (b) and (d) spectra of doubly and triply charged TSTEPQyQPGENL (y indicates phosphorylated tyrosine); * indicates the precursor ion 


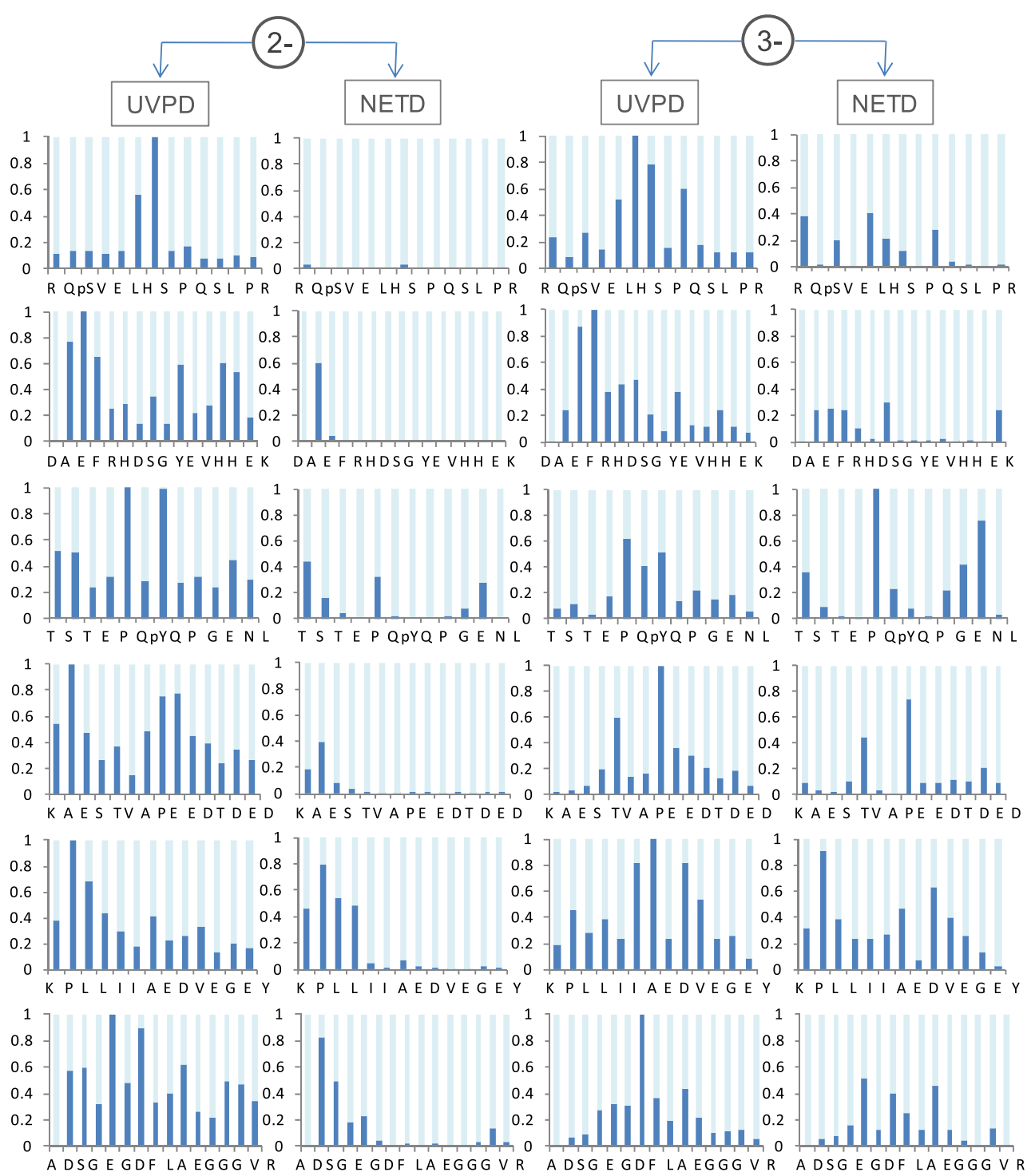

Figure 3. Sequence coverage distribution comparison between UVPD and NETD of doubly and triply deprotonated peptide anions. The product ion signals were plotted for summed $a / x$ pairs per backbone cleavage. For each peptide, ion signals were normalized to the highest $a / x$ pair between the UVPD and NETD methods

and LC-MS/UVPD in addition to positive ion mode LC-MS/ CID experiments were undertaken for tryptic digests of a model protein mixture containing bovine $\alpha$-casein, $\beta$-casein, $\beta$-lactoglobulin $\mathrm{A}, \mathrm{BSA}$, ribonuclease $\mathrm{A}$, and chicken lysozyme $\mathrm{C}$ with automated database searching via MassMatrix. These proteins were chosen for three reasons: they are well characterized model systems, $\alpha$-casein and $\beta$-casein are highly phosphorylated, and the theoretical isoelectric points (based on amino acid content) of these proteins range from $\sim 4.8$ for $\beta$-lactoglobulin to $\sim 9.3$ for lysozyme $\mathrm{C}$ and thus should not bias results for either positive or negative mode analyses. Table 2 summarizes the results obtained from the NETD, UVPD, and CID data sets. UVPD produced overall higher sequence coverage percentages and greater numbers of unique peptides compared to that of NETD. This outcome can be attributed to the ability of UVPD to
Table 1. Sequence Coverage Comparison of NETD and UVPD of Peptide anions. The Percentages were Calculated from $a$ - and $x$-type Ions with $\mathrm{S} / \mathrm{N} \geq$ 3, and Represent the Number of Observed Backbone Cleavages divided by the Total Possible Backbone Cleavages per Peptide

\begin{tabular}{|c|c|c|c|c|}
\hline \multirow[t]{3}{*}{ Peptide sequence } & \multicolumn{4}{|c|}{$\%$ Sequence coverage } \\
\hline & \multicolumn{2}{|c|}{$[\mathrm{M}-2 \mathrm{H}]^{2-}$} & \multicolumn{2}{|c|}{$[\mathrm{M}-3 \mathrm{H}]^{3-}$} \\
\hline & NETD & UVPD & NETD & UVPD \\
\hline RQsVELHSPQSLPR & 15 & 100 & 77 & 100 \\
\hline DAEFRHDSGYEVHHEK & 13 & 100 & 60 & 100 \\
\hline TSTEPQyQPGENL & 50 & 100 & 75 & 100 \\
\hline KAESTVAPEEDTDED & 43 & 100 & 93 & 100 \\
\hline KPLLIIAEDVEGEY & 62 & 100 & 100 & 100 \\
\hline ADSGEGDFLAEGGGVR & 40 & 100 & 93 & 100 \\
\hline
\end{tabular}


Table 2. MassMatrix Comparison of Negative Mode NETD and UVPD and Positive Mode CID LC-MS/MS Experiments

\begin{tabular}{|c|c|c|c|c|c|c|}
\hline \multirow[t]{2}{*}{ Protein } & \multicolumn{3}{|c|}{$\%$ Sequence } & \multicolumn{3}{|c|}{ Unique peptides } \\
\hline & NETD & UVPD & CID & NETD & UVPD & CID \\
\hline BSA & 51 & 58 & 70 & 26 & 33 & 51 \\
\hline$\alpha \mathrm{S} 1$-casein & 35 & 43 & 32 & $9(4 / 1)^{*}$ & $12(4 / 1)^{*}$ & $9(2 / 1)^{*}$ \\
\hline$\alpha \mathrm{S} 2$-casein & 38 & 32 & 31 & $6(4 / 4) *$ & $9(4 / 4)^{*}$ & $12(3 / 2)^{*}$ \\
\hline$\beta$-Lactoglobulin A & 64 & 56 & 48 & 7 & 8 & 11 \\
\hline$\beta$-Casein & 15 & 24 & 25 & $2(1 / 1)^{*}$ & $6(1 / 1)^{*}$ & $6(2 / 1)^{*}$ \\
\hline Lysozyme C & 24 & 43 & 81 & 2 & 4 & 10 \\
\hline Ribonuclease A & 13 & 42 & 52 & 1 & 3 & 5 \\
\hline
\end{tabular}

*Unique peptides (phosphopeptides/phosphorylation sites)

efficiently sequence both lower and higher charge states with rapid activation times $(4 \mathrm{~ms})$. In contrast, NETD in general yields the best results for higher charged states $(n \geq 3)$, and uses fairly modest activation times $(250 \mathrm{~ms})$, which can limit the number of MS/MS spectra collected. As expected, due to higher signal intensities of tryptic peptides (ones that contain at least two protonation sites) in the positive mode, CID generally yielded the most unique peptide identifications and sequence coverage compared to both negative mode MS/MS methods, except for three of the model proteins, $\alpha \mathrm{S} 1$-casein, $\alpha$ S2-casein, and $\beta$-lactoglobulin A. Both UVPD and NETD, however, often outperformed CID for sequencing and identifying acidic peptide species. UVPD identified a total of nine phosphopeptides corresponding to six unique phosphorylation sites, and NETD also identified nine phosphopeptides corresponding to six unique phosphorylation sites. CID identified seven phosphopeptides corresponding to four unique phosphorylation sites. The Venn diagram in Figure 4 shows the overlap in unique peptides identified in the negative mode by NETD and UVPD and in the positive mode by CID for the protein mixture. A significant overlap in peptide matches was observed between NETD and UVPD with $83 \%$ of those

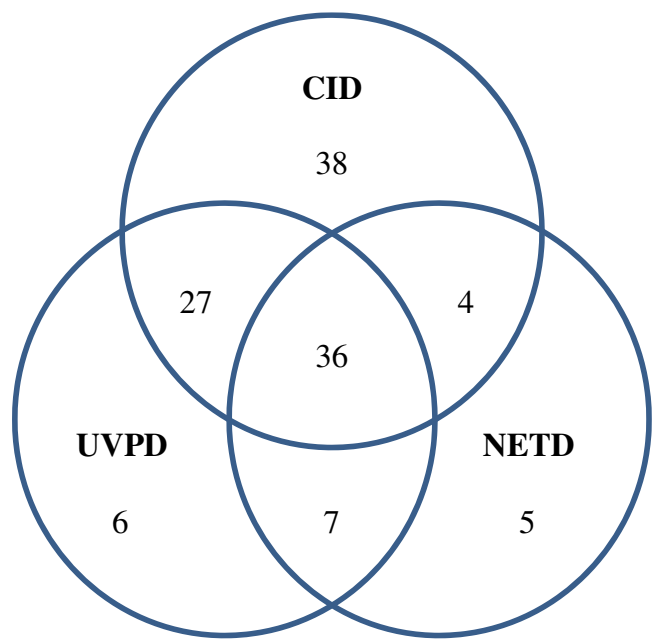

Figure 4. Venn diagram comparing the overlap in unique peptides identified in the negative mode by NETD and UVPD and in the positive mode by CID
Table 3. MassMatrix Comparison of NETD/CID and UVPD/CID Combined Positive and Negative Mode Data Sets

\begin{tabular}{lccllll}
\hline \multirow{2}{*}{ Protein } & \multicolumn{2}{c}{$\%$ Sequence } & & \multicolumn{2}{c}{ Unique peptides } \\
\cline { 2 - 3 } \cline { 6 - 7 } \cline { 5 - 6 } & NETD/CID & UVPD/CID & & NETD/CID & UVPD/CID \\
\hline BSA & 77 & 73 & & 54 & 54 \\
$\alpha$ S1-casein & 47 & 46 & & $13(4 / 1)^{*}$ & $13(4 / 1)^{*}$ \\
$\alpha$ S2-casein & 46 & 36 & & $14(4 / 4)^{*}$ & $15(5 / 4)^{*}$ \\
$\beta$-Lactoglobulin A & 63 & 63 & & 14 & 13 \\
$\beta$-Casein & 25 & 29 & & $6(2 / 1)^{*}$ & $7(2 / 1)^{*}$ \\
Lysozyme C & 81 & 81 & & 10 & 10 \\
Ribonuclease A & 52 & 52 & & 5 & 5 \\
\hline
\end{tabular}

*Unique peptides (phosphopeptides/phosphorylation sites)

identified by NETD also identified by UVPD. UVPD and NETD produced 13 and 12 unique peptides, respectively, which were not identified by CID in positive mode.

Lastly, database searches were undertaken by combining the spectral files for UVPD and positive CID as well as NETD and positive CID to investigate in more detail the overlap between traditional CID and the negative mode methods; these results are illustrated in Table 3. In all cases except for lysozyme $\mathrm{C}$ and ribonuclease $\mathrm{A}$, the combined searches significantly increased the sequence coverage percentages and unique peptide identifications over that obtained by CID alone. The combined NETD/CID searches outperformed the UVPD/CID searches for BSA, but underperformed for $\beta$-casein. However, even these search outcomes were fairly similar (i.e., the number of unique peptides and sequence coverage percentages were close if not identical). The integrated NETD/CID and UVPD/CID results were similar for most of the other proteins. The variations that were seen between the two different searching strategies are attributed to the differences in optimum ion charge state for each MS/MS method and the corresponding population of charge states generated for the tryptic peptides of each protein (as described in previous sections). To investigate this hypothesis in more quantifiable detail, the

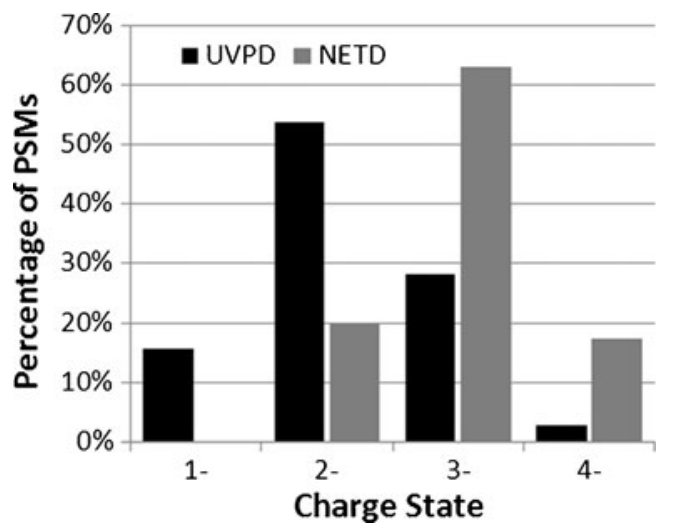

Figure 5. Charge state distribution of peptide spectral matches (PSMs) produced by NETD and UVPD for a protein mixture. The total number of PSMs for NETD and UVPD are 259 and 578, respectively 
charge state distributions of the peptide spectral matches (PSMs) produced by NETD and UVPD are tabulated in Figure 5. For UVPD, the charge state distribution $(\bar{x}=-2.1$, $\mathrm{s}=0.72, n=578)$ is centered about the 2- charge state which corresponded to $54 \%$ of the PSMs. In contrast, the charge state distribution for NETD $(\bar{x}=-2.9, \mathrm{~s}=0.60, n=259)$ is centered about the 3-charge state, which corresponded to $63 \%$ of the PSMs. These differences in peptide preference highlight the complementary nature of the two MS/MS methods for peptide anions and are responsible for many of the observed variations in the acidic peptide identifications.

\section{Conclusion}

UVPD and NETD were compared with respect to sequence coverage distributions (a measure of product ion signals across the peptide backbone), sequence coverage percentages, backbone cleavage specificity, and both LC-MS/MS and automated database searching performance metrics for analysis of peptide anions. The substantially shorter activation times and compatibility with a wider range of charge states allowed the collection of a greater number of MS/MS spectra during a typical a LC gradient for UVPD compared with NETD. However, the spectra that were collected for NETD were generally from peptide species that contained several acidic residues and/or acidic post-translational modifications - ones that are more difficult for traditional positive mode CID to efficiently and accurately elucidate. Importantly, when combined with positive mode CID, both UVPD and NETD significantly increased the sequence coverage percentages and unique peptide identifications over that of just CID alone, demonstrating their utility and potential for characterizing acidic proteomes.

\section{Acknowledgments}

J.S.B. acknowledges funding from the NIH (R21GM099028) and the Welch Foundation (F-1155). H.X. acknowledges funding from the NIH (UL1 RR-024989, PI: Dr. Pamela B. Davis).

\section{References}

1. Hunt, D.F., Buko, A.M., Ballard, J.M., Shabanowitz, J., Giordani, A.B.: Sequence analysis of polypeptides by collision activated dissociation on a triple quadrupole mass spectrometer. Biol. Mass Spectrom. 8, $397-$ 408 (1981)

2. McLuckey, S.: Principles of collisional activation in analytical mass spectrometry. J. Am. Soc. Mass Spectrom. 3, 599-614 (1992)

3. Eng, J.K., McCormack, A.L., Yates III, J.R.: An approach to correlate tandem mass spectral data of peptides with amino acid sequences in a protein database. J. Am. Soc. Mass Spectrom. 5, 976-989 (1994)

4. Perkins, D.N., Pappin, D.J.C., Creasy, D.M., Cottrell, J.S.: Probabilitybased protein identification by searching sequence databases using mass spectrometry data. Electrophoresis 20, 3551-3567 (1999)

5. de Godoy, L.M.F., Olsen, J.V., Cox, J., Nielsen, M.L., Hubner, N.C., Frohlich, F., Walther, T.C., Mann, M.: Comprehensive mass-spectrometry-based proteome quantification of haploid versus diploid yeast. Nature 455, 1251-1254 (2008)
6. Zubarev, R.A., Kelleher, N.L., McLafferty, F.W.: Electron capture dissociation of multiply charged protein cations. A nonergodic process. J. Am. Chem. Soc. 120, 3265-3266 (1998)

7. Kelleher, N.L., Zubarev, R.A., Bush, K., Furie, B., Furie, B.C., McLafferty, F.W., Walsh, C.T.: Localization of labile post-translational modifications by electron capture dissociation: the case of $\gamma$-carboxyglutamic acid. Anal. Chem. 71, 4250-4253 (1999)

8. Zubarev, R.A., Horn, D.M., Fridriksson, E.K., Kelleher, N.L., Kruger, N.A., Lewis, M.A., Carpenter, B.K., McLafferty, F.W.: Electron capture dissociation for structural characterization of multiply charged protein cations. Anal. Chem. 72, 563-573 (2000)

9. Syka, J.E.P., Coon, J.J., Schroeder, M.J., Shabanowitz, J., Hunt, D.F.: Peptide and protein sequence analysis by electron transfer dissociation mass spectrometry. PNAS 101, 9528-9533 (2004)

10. Coon, J.J., Syka, J.E.P., Schwartz, J.C., Shabanowitz, J., Hunt, D.F.: Anion dependence in the partitioning between proton and electron transfer in ion/ion reactions. Int. J. Mass Spectrom. 236, 33-42 (2004)

11. Good, D.M., Wirtala, M., McAlister, G.C., Coon, J.J.: Performance characteristics of electron transfer dissociation mass spectrometry. Mol. Cell. Proteom. 6, 1942-1951 (2007)

12. Bowers, W.D., Delbert, S.S., Hunter, R.L., McIver, R.T.: Fragmentation of oligopeptide ions using ultraviolet laser radiation and Fourier transform mass spectrometry. J. Am. Chem. Soc. 106, 7288-7289 (1984)

13. Little, D.P., Speir, J.P., Senko, M.W., O'Connor, P.B., McLafferty, F.W.: Infrared multiphoton dissociation of large multiply charged ions for biomolecule sequencing. Anal. Chem. 66, 2809-2815 (1994)

14. Hofstadler, S.A., Sannes-Lowery, K.A., Griffey, R.H.: Infrared multiphoton dissociation in an external ion reservoir. Anal. Chem. 71, 20672070 (1999)

15. Wilson, J.J., Brodbelt, J.S.: Infrared multiphoton dissociation for enhanced de novo sequence interpretation of $\mathrm{N}$-terminal sulfonated peptides in a quadrupole ion trap. Anal. Chem. 78, 6855-6862 (2006)

16. Morgan, J.W., Hettick, J.M., Russell, D.H.: In: Biological Mass Spectrometry; Academic Press; Vol. 402, pp. 186-209 (2005)

17. Kim, T.-Y., Thompson, M.S., Reilly, J.P.: Peptide photodissociation at $157 \mathrm{~nm}$ in a linear ion trap mass spectrometer. Rapid Commun. Mass Spectrom. 19, 1657-1665 (2005)

18. Ly, T., Julian, R.R.: Ultraviolet photodissociation: developments towards applications for mass-spectrometry-based proteomics. Angew. Chem. Int. Ed. 48, 7130-7137 (2009)

19. Reilly, J.P.: Ultraviolet photofragmentation of biomolecular ions. Mass Spectrom. Rev. 28, 425-447 (2009)

20. Ly, T., Julian, R.R.: Residue-specific radical-directed dissociation of whole proteins in the gas phase. J. Am. Chem. Soc. 130, 351-358 (2008)

21. Morgan, J.W., Russell, D.H.: Comparative studies of 193-nm photodissociation and TOF-TOFMS analysis of bradykinin analogues: the effects of charge site(s) and fragmentation timescales. J. Am. Soc. Mass Spectrom. 17, 721-729 (2006)

22. Thompson, M.S., Cui, W., Reilly, J.P.: Factors that impact the vacuum ultraviolet photofragmentation of peptide ions. J. Am. Soc. Mass Spectrom. 18, 1439-1452 (2007)

23. Kim, T.-Y., Reilly, J.P.: Time-resolved observation of product ions generated by $157 \mathrm{~nm}$ photodissociation of singly protonated phosphopeptides. J. Am. Soc. Mass Spectrom. 20, 2334-2341 (2009)

24. Madsen, J.A., Boutz, D.R., Brodbelt, J.S.: Ultrafast ultraviolet photodissociation at $193 \mathrm{~nm}$ and its applicability to proteomic workflows. $J$. Proteome Res. 9, 4205-4214 (2010)

25. Shin, Y.S., Moon, J.H., Kim, M.S.: Observation of phosphorylation site-specific dissociation of singly protonated phosphopeptides. J. Am. Soc. Mass Spectrom. 21, 53-59 (2010)

26. Brodbelt, J.S.: Shedding light on the frontier of photodissociation. $J$. Am. Soc. Mass Spectrom. 22, 197-206 (2001)

27. Time-resolved photodissociation of singly protonated peptides with an arginine at the N-terminus: a statistical interpretation. J. Am. Soc. Mass Spectrom. 19, 645-655 (2008)

28. Janek, K., Wenschuh, H., Bienert, M., Krause, E.: Phosphopeptide analysis by positive and negative ion matrix-assisted laser desorption/ ionization mass spectrometry. Rapid Commun. Mass Spectrom. 15, 1593-1599 (2001)

29. Gunawardena, H.P., Emory, J.F., McLuckey, S.A.: Phosphopeptide anion characterization via sequential charge inversion and electrontransfer dissociation. Anal. Chem. 78, 3788-3793 (2006) 
30. Ewing, N., Cassady, C.: Dissociation of multiply charged negative ions for hirudin (54-65), fibrinopeptide B, and insulin A (oxidized). J. Am. Soc. Mass Spectrom. 12, 105-116 (2001)

31. Bowie, J.H., Brinkworth, C.S., Dua, S.: Collision-induced fragmentations of the $(\mathrm{M}-\mathrm{H})^{-}$parent anions of underivatized peptides: An aid to structure determination and some unusual negative ion cleavages. Mass Spectrom. Rev. 21, 87-107 (2002)

32. Steinborner, S.T., Bowie, J.H.: The negative ion mass spectra of [M $\mathrm{H}]^{-}$ions derived from caeridin and dynastin peptides. Internal backbone cleavages directed through Asp and Asn residues. Rapid Commun. Mass Spectrom. 11, 253-258 (1997)

33. Jensen, O.N.: Interpreting the protein language using proteomics. Nat. Rev. Mol. Cell. Biol. 7, 391-403 (2006)

34. Schwartz, R., Ting, C.S., King, J.: Whole proteome pI values correlate with subcellular localizations of proteins for organisms within the three domains of life. Genome Res. 11, 703-709 (2001)

35. Knight, C.G., Kassen, R., Hebestreit, H., Rainey, P.B.: Global analysis of predicted proteomes: functional adaptation of physical properties. Proc. Nat. Acad. Sci. U.S.A. 101, 8390-8395 (2004)

36. Weiller, G.F., Caraux, G., Sylvester, N.: The modal distribution of protein isoelectric points reflects amino acid properties rather than sequence evolution. Proteomics 4, 943-949 (2004)

37. Kiraga, J., Mackiewicz, P., Mackiewicz, D., Kowalczuk, M., Biecek, P., Polak, N., Smolarczyk, K., Dudek, M., Cebrat, S.: The relationships between the isoelectric point and: length of proteins, taxonomy and ecology of organisms. BMC Genom. 8, 163 (2007)

38. Kjeldsen, F., Silivra, O.A., Ivonin, I.A., Haselmann, K.F., Gorshkov, M., Zubarev, R.A.: $\mathrm{C} \alpha-\mathrm{C}$ Backbone Fragmentation Dominates in Electron Detachment Dissociation of Gas-Phase Polypeptide Polyanions. Chem. Eur. J. 11, 1803-1812 (2005)

39. Kjeldsen, F., Horning, O.B., Jensen, S.S., Giessing, A.M.B., Jensen, O.N.: Towards liquid chromatography time-scale peptide sequencing and characterization of post-translational modifications in the negativeion mode using electron detachment dissociation tandem mass spectrometry. J. Am. Soc. Mass Spectrom. 19, 1156-1162 (2008)

40. Larraillet, V., Vorobyev, A., Brunet, C., Lemoine, J., Tsybin, Y.O., Antoine, R., Dugourd, P.: Comparative dissociation of peptide polyanions by electron impact and photo-induced electron detachment. J. Am. Soc. Mass Spectrom. 21, 670-680 (2010)

41. Cook, S., Jackson, G.: Metastable atom-activated dissociation mass spectrometry of phosphorylated and sulfonated peptides in negative ion mode. J. Am. Soc. Mass Spectrom. 22, 1088-1099 (2011)

42. Huzarska, M., Ugalde, I., Kaplan, D.A., Hartmer, R., Easterling, M.L., Polfer, N.C.: Negative electron transfer dissociation of deprotonated phosphopeptide anions: choice of radical cation reagent and competition between electron and proton transfer. Anal. Chem. 82, 2873-2878 (2010)

43. Coon, J.J., Shabanowitz, J., Hunt, D.F., Syka, J.E.P.: Electron transfer dissociation of peptide anions. J. Am. Soc. Mass Spectrom. 16, 880-882 (2005)

44. Madsen, J.A., Kaoud, T.S., Dalby, K.N., Brodbelt, J.S.: 193-nm photodissociation of singly and multiply charged peptide anions for acidic proteome characterization. Proteomics 11, 1329-1334 (2011)

45. Antoine, R., Joly, L., Tabarin, T., Broyer, M., Dugourd, P., Lemoine, J.: Photo-induced formation of radical anion peptides. Electron photodetachment dissociation experiments. Rapid Commun. Mass Spectrom 21, 265-268 (2007)

46. McAlister, G.C., Russell, J.D., Rumachik, N.G., Hebert, A.S., Syka, J.E.P., Geer, L.Y., Westphall, M.S., Pagliarini, D.J., Coon, J.J.: Analysis of the acidic proteome with negative electron-transfer dissociation mass spectrometry. Anal. Chem. 84, 2875-2882 (2012)

47. Madsen, J.A., Xu, H., Horton, A.P., Boutz, D.R., Kaoud, T.S., Dalby, K.N., Brodbelt, J.S.: A flexible searching algorithm for acidic proteome characterization by UVPD of peptide anions, unpublished (submitted)

48. Xu, H., Freitas, M.: A mass accuracy sensitive probability based scoring algorithm for database searching of tandem mass spectrometry data. BMC Bioinformatics 8, 133 (2007)

49. Xu, H., Zhang, L., Freitas, M.A.: Identification and characterization of disulfide bonds in proteins and peptides from tandem MS data by use of the MassMatrix MS/MS search engine. J. Proteome Res. 7, 138-144 (2008)

50. Xu, H., Hsu, P.-H., Zhang, L., Tsai, M.-D., Freitas, M.A.: Database search algorithm for identification of intact cross-links in proteins and peptides using tandem mass spectrometry. J. Proteome Res. 9, 33843393 (2010) 